\title{
Magnetoelectric effect in conduction-electron spin resonance in zinc-blende semiconductors
}

\author{
Yang-Fang Chen \\ Department of Physics, National Taiwan University, Taipei, Taiwan, Republic of China
}

(Received 14 February 1989)

\begin{abstract}
We propose a new phenomenon in zinc-blende semiconductors, the magnetoelectric effect, which occurs when there exist simultaneously electric-dipole- and magnetic-dipole-induced conductionelectron spin resonance. We point out that this effect can lead to an anomalous dependence of the spin-resonance intensity on the sign of either the dc magnetic field $\mathbf{B}_{0}$ or the wave vector $\mathbf{q}$ of the photon. The effect also provides an elegant way of determining the parameter which causes the electric-dipole-induced conduction-electron spin resonance.
\end{abstract}

A crystal placed in an applied electric field may experience a magnetization, and placed in a magnetic field may experience an electric polarization. Since Curie ${ }^{1}$ first proposed this magnetoelectric field, it has been studied extensively, ${ }^{2}$ but so far the research has been restricted only to magnetic crystals.

In the present Report we wish to propose the existence of the magnetoelectric effect in zinc-blende semiconductors, which can occur when electric-dipole- and magnetic-dipole-induced conduction-electron spin resonance exist simultaneously. This theory correctly predicts the experimental observations ${ }^{3-5}$ that in the parallel Voigt geometry $\left(\mathbf{E} \| \mathbf{B}_{0} \perp \mathbf{q}\right)$ the spin-resonance spectra reveal an anomalous dependence on the sign of either the dc magnetic field $\mathbf{B}_{0}$ or the wave vector $\mathbf{q}$ of the photon.

The perturbation operator of an electromagnetic wave with a vector potential $\mathbf{A}$ acting on an electron is given by

$$
H^{\prime}=\frac{e}{c m} \mathbf{P} \cdot \mathbf{A}+\frac{g \beta_{0}}{2} \boldsymbol{\sigma} \cdot \boldsymbol{\nabla} \times \mathbf{A},
$$

where $\mathbf{P}$ is the momentum operator, $g$ is the $g$ factor of the electron, $\beta_{0}=e h / 2 m_{0} c$ is the Bohr magneton, $m_{0}$ is the mass of the free electron, and $\sigma=\left(\sigma_{x}, \sigma_{y}, \sigma_{z}\right)$ is the Pauli matrix. The perturbed wave function $\Psi$ can be determined from the dynamical equation of quantum mechanics

$$
i \hbar \frac{\partial \Psi}{\partial t}=\left(H_{0}+H^{\prime}\right) \Psi
$$

in which $H_{0}$ is the unperturbed Hamiltonian. For any observable quantity $Q$ (such as the electric dipole or magnetic dipole moment) is governed by its expectation value, which can be written as

$$
Q=\int \Psi^{*} Q \Psi d \mathbf{r}
$$

It can be shown ${ }^{6}$ that the induced dipole moment $\mathbf{P}_{1}$ has the form

$$
\begin{aligned}
\mathbf{P}_{1}=2 \sum_{m} & \left(\frac{E_{m n}}{E_{m n}^{2}-E^{2}-i \hbar \omega \Gamma_{m n}^{\alpha}} \operatorname{Re}(\langle n|\mathbf{P}| m\rangle\langle m|\mathbf{P}| n\rangle) \cdot \mathbf{E}-\frac{h}{E_{m n}^{2}-E^{2}-i \hbar \omega \Gamma_{m n}^{\beta}} \operatorname{Im}(\langle n|\mathbf{P}| m\rangle\langle m|\mathbf{P}| n\rangle) \cdot \dot{\mathbf{E}}\right. \\
& \left.+\frac{E_{m n}}{E_{m n}^{2}-E^{2}-i \hbar \omega \Gamma_{m n}^{\gamma}} \operatorname{Re}(\langle n|\mathbf{P}| m\rangle\langle m|\mathbf{m}| n\rangle) \cdot \mathbf{H}-\frac{h}{E_{m n}^{2}-E^{2}-i \hbar \omega \Gamma_{m n}^{\delta}} \operatorname{Im}(\langle n|\mathbf{p}| m\rangle\langle m|\mathbf{m}| n\rangle) \cdot \dot{\mathbf{H}}\right) .
\end{aligned}
$$

Here; we have neglected the contribution of the electric quadrupole moment. In the above equation the following abbreviations have been introduced: $E_{m n}$ is the energy difference between state $|m\rangle$ and state $|n\rangle, E=\hbar \omega$ represents the quantum energy associated with the electromagnetic (EM) wave, $\Gamma_{m n}$ measures the strength of the damping term, and $\mathbf{m}=\frac{1}{2} g \beta_{0} \sigma+\left(e / 2 m_{0} c\right) \mathbf{r} \times \mathbf{p}, \mathbf{E}$ and $H$ are, respectively, the electric and magnetic fields, and $\dot{\mathbf{E}}$ and $\dot{H}$ are time derivatives of $\mathbf{E}$ and $\mathbf{H}$, respectively. The corresponding expression for the induced magnetic dipole moment $\mathbf{m}_{1}$ is

$$
\begin{aligned}
\mathbf{m}_{1}=2 \sum_{m} & \frac{E_{m n}}{E_{m n}^{2}-E^{2}-i \hbar \omega \Gamma_{m n}^{\gamma}} \\
& \times \operatorname{Re}(\langle n|\mathbf{m}| m\rangle\langle m|\mathbf{P}| n\rangle) \cdot \mathbf{E} \\
& -\frac{h}{E_{m n}^{2}-E^{2}-i \hbar \omega \Gamma_{m n}^{\delta}} \\
& \quad \times \operatorname{Im}(\langle n|\mathbf{m}| m\rangle\langle m|\mathbf{P}| n\rangle) \cdot \dot{\mathbf{E}}] .
\end{aligned}
$$

In Eq. (5) the contribution of the magnetic field, which is 
proportional to $\langle n|\mathbf{m}| m\rangle\langle m|\mathbf{m}| n\rangle$, has been neglected because it is much smaller than the contribution of the electric field. Equations (4) and (5) can be written in a more compact form as

$$
\mathbf{P}_{1}=\overleftrightarrow{\alpha} \cdot \mathbf{E}-\overleftrightarrow{\beta} \cdot \dot{\mathbf{E}}+\overleftrightarrow{\gamma} \cdot \mathbf{H}-\overleftrightarrow{\delta} \cdot \dot{\mathbf{H}}
$$

and

$$
\mathbf{m}_{1}=\overleftrightarrow{\gamma} \cdot \mathbf{E}+\overleftrightarrow{\delta} \cdot \dot{\mathbf{E}}
$$

The corresponding matrix elements for $\overleftrightarrow{\alpha}, \overleftrightarrow{\beta}, \overleftrightarrow{\gamma}$, and $\overleftrightarrow{\delta}$ can be easily found out. For instance,

$\alpha_{i j}=2 \sum_{m} \frac{E_{m n}}{E_{m n}^{2}-E^{2}-i \hbar \omega \Gamma_{m n}^{\alpha}} \operatorname{Re}\left(\left\langle n\left|P_{i}\right| m\right\rangle\left\langle m\left|P_{j}\right| n\right\rangle\right)$.

Thus from Eqs. (6) and (7) we can see that the magnetoelectric effect appears as long as the electric dipole and the magnetic dipole can simultaneously induce the transition of electron from state $|n\rangle$ to state $|m\rangle$.

The above derivation is quite general and is true for any substance. Let us now examine this effect in the conduction-electron spin resonance in zinc-blende semiconductors. It is well known that under certain circumstances in the presence of spin-orbit interaction, transitions which involve a change in the spin state of the carrier can be excited by electric dipole radiation. It has been shown theoretically and experimentally that electric dipole spin-flip transition can arise from three possible sources: (1) inversion asymmetry, (2) nonparabolicity, and (3) warping. 4,5,7-11 Experimental studies ${ }^{4,5}$ have shown that the dominant mechanism is the inversion asymmetry. In the following discussion we will restrict ourselves to the electric dipole spin resonance induced by this mechanism. Thus it is possible that both the electric dipole and the magnetic dipole can induce electron-flip transition, and hence the magnetoelectric effect will appear in the zinc-blende semiconductors. We shall now examine this effect in some principal experimental configurations.

Ordinary Voigt geometry (OV): This configuration refers to where the propagation of the wave is perpendicular to the dc magnetic field $\mathbf{B}_{0}$, and the electric field $\mathbf{E}$ is parallel to $B_{0}$. It has been shown ${ }^{4,5}$ that in OV geometry the transition matrix element of electric dipole spin resonance is in phase with that of magnetic dipole spin resonance (both are real). Thus Eqs. (6) and (7) are now reduced to

$$
\begin{aligned}
& \mathbf{P}_{1}=\overleftrightarrow{\alpha} \cdot \mathbf{E}-\overleftrightarrow{\beta} \cdot \dot{\mathbf{E}}+\overleftrightarrow{\gamma} \cdot \mathbf{H} \\
& \mathbf{m}_{1}=\overleftrightarrow{\gamma} \cdot \mathbf{E}
\end{aligned}
$$

In this case, the dissipated energy can be obtained by the expression

$$
P=\frac{1}{8 \pi} \omega \mathbf{E} \cdot \overleftrightarrow{\alpha^{\prime \prime}} \cdot \mathbf{E}^{*}+\omega \mathbf{E} \cdot \overbrace{}^{\prime \prime} \cdot \mathbf{H}^{*},
$$

where $\overleftrightarrow{\alpha^{\prime \prime}}$ and $\overleftrightarrow{\gamma^{\prime \prime}}$ are the imaginary parts of $\overleftrightarrow{\alpha}$ and $\overleftrightarrow{\gamma}$, respectively. In addition to the Ohmic power dissipation energy (the first term), we have the energy absorption due to the magnetoelectric effect (the second term). Following the derivation of Rashba and Sheka, ${ }^{4,5,7}$ the term induced by magnetoelectric effect, $P_{\mathrm{ME}}$, can be written as

$$
P_{\mathrm{ME}} \propto f(\theta, \phi) B_{0} q,
$$

where $\theta$ and $\phi$ are the polar and azimuthal angle of the dc magnetic field with respect to the crystal axes, respectively, and $q$ is the magnitude of the wave vector of the photon. It can be used to explain the anomalous dependence of the conduction-electron spin-resonance intensity on the sign of either the dc magnetic field $\mathbf{B}_{0}$ or the wave vector $\mathbf{q}$, which have been observed previously. ${ }^{3-5}$

In order to compare the theoretical and experimental results, we need the exact expression for the corresponding absorption coefficient as a function of orientation of $\mathbf{B}_{0}$ which has been derived by Chen et al. ${ }^{5}$ As an illustration, we will only show the case of $\mathbf{B}_{0}$ in the (112) plane. The expression can be written as

$$
\begin{aligned}
\alpha=\frac{8 \pi C N}{n w h r}[ & {\left[\frac{3}{4} \frac{\delta_{0} e^{2} B_{0}}{c^{2} h^{2}}\right]^{2}\left\{[\cos (2 \phi) \sin (2 \theta)]^{2}+\left[\sin (2 \phi) \sin \theta\left(2 \cos ^{2} \theta-\sin ^{2} \theta\right)\right]^{2}\right\} } \\
+ & \frac{3}{4 \sqrt{6}} \frac{\delta_{0} e^{2} B_{0}}{c^{2} h^{2}} g \beta_{0} q\{\cos (2 \phi) \sin (2 \theta)[\cos \theta(\cos \phi-\sin \phi)-2 \sin \theta] \\
& \left.\left.\quad+\sin (2 \phi) \sin \theta\left(2 \cos ^{2} \theta-\sin ^{2} \theta\right)(\cos \phi+\sin \phi)\right\}+\left[\frac{g \beta_{0} q}{2}\right]^{2}\right],
\end{aligned}
$$

where $N$ is the free-carrier concentration, $n$ is the refractive index, $r$ is a phenomenological broadening constant, and $\delta_{0}$ is the inversion asymmetry parameter.

Figure 1 shows the spin-resonance absorption coefficients as a function of orientation of $B_{0}$ in the (112) plane. The measurements were performed on InSb at $118.8 \mu \mathrm{m}$ and $4.5 \mathrm{~K}^{5}$ Note the strong angular dependence of the data, and the absorption coefficients corresponding to opposite field directions, indicated by solid and open circles, respectively. The theoretical curves are obtained by adjusting the value or $\sigma_{0}$, with the solid and dashed curves drawn for opposite directions of $\boldsymbol{B}_{0}$. The obtained inversion asymmetry parameter $\delta_{0}=56$ a.u. (atomic unit), or $3.6 \times 10^{-34} \mathrm{erg} \mathrm{cm}^{3}$. The agreement between experimental and theoretical angular dependence of the spin-resonance absorption coefficient provides an excellent demonstration of the existence of magnetoelectric effect in zinc-blende semiconductors. 


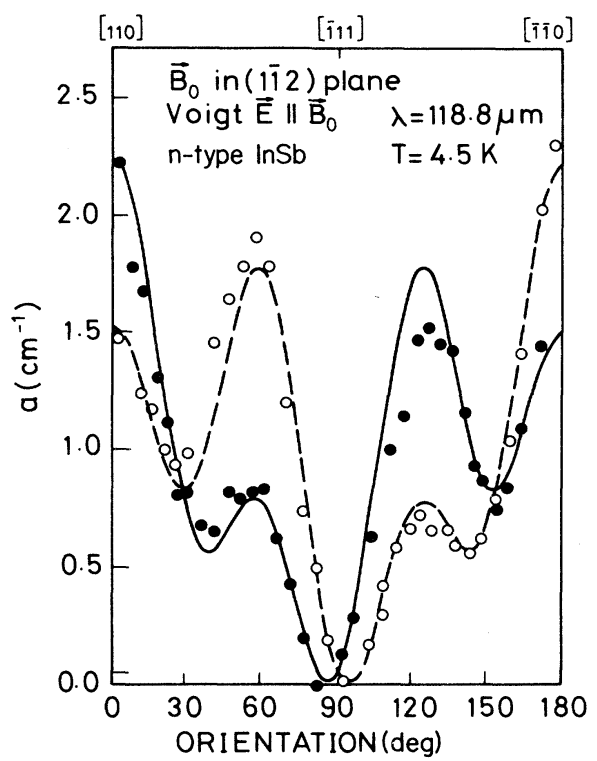

FIG. 1. Spin-resonance absorption coefficient of conduction electrons in $n$-type $\mathrm{InSb}$ as a function of $\mathbf{B}_{0}$ in the (112) plane. Solid and open circles correspond to opposite signs of $\mathbf{B}_{0}$, respectively. The solid and dashed curves are the theoretical angular dependences fitted to the experimental data for opposite field directions. The data were taken at $118.8 \mu \mathrm{m}$ and $4.5 \mathrm{~K}$.

Faraday geometry: This configuration refers to the propagation of the wave parallel to $\mathbf{B}_{0}$. There are two normal modes which can be supported by the medium, the cyclotron-resonance-active (CRA) polarization and the cyclotron-resonance-inactive (CRI) polarization. For the CRA polarization there is no magnetic dipole transition because $g<0$, and the magnetoelectric effect will therefore vanish. In the CRI polarization, the transition matrix element of electric dipole spin resonance is always out of phase (one is imaginary while the other one is real). ${ }^{4,5}$ Thus Eqs. (6) and (7) have the form

$$
\begin{aligned}
& \mathbf{P}_{1}=\overleftrightarrow{\alpha} \cdot \mathbf{E}-\overleftrightarrow{\beta} \cdot \dot{\mathbf{E}}-\overleftrightarrow{\delta} \cdot \dot{\mathbf{H}}, \\
& \mathbf{m}_{1}=\overleftrightarrow{\delta} \cdot \dot{\mathbf{E}} .
\end{aligned}
$$

Now, the dissipated energy is only caused by the usual Ohmic power dissipation. Thus the conduction-electron spin-resonance intensity should not depend on the sign of either $\mathbf{B}_{0}$ or $\mathbf{q}$, which is consistent with the experimental results. ${ }^{3-5}$

In summary, we have proposed that the magnetoelectric effect exists in conduction-electron spin resonance in zinc-blende semiconductors. It can explain the peculiar phenomena occurring in the spin-flip transition in zincblende semiconductors, such as the anomalous dependence of the resonance intensity on the direction of $\mathbf{B}_{0}$ and $\mathbf{q}$.

Here we have only analyzed crystals of the zinc-blende type. Extending these considerations to other types of accentric crystals (e.g., wurtzite) should lead to similar results. In view of the recent observation of electric dipole spin resonance in $\mathrm{Cd}_{1-x} \mathrm{Mn}_{x} \mathrm{Se}{ }^{12}$ such analysis would be useful and timely.

We would like to thank Professor J. K. Furdyna and Dr. Y. H. Chang for enlightening discussions.
${ }^{1}$ P. Curie, J. Phys. (Paris) 3 Serie, III, 393 (1894).

${ }^{2}$ T. H. O'Dell, The Electrodynamics of Magnetoelectric Media, edited by E. P. Wohlfarth (North-Holland, Amsterdam, 1970), Vol. XI.

${ }^{3}$ M. Dobrowolska, Y. F. Chen, J. K. Furdyna, and S. Rodriguez, Phys. Rev. Lett. 51, 134 (1983).

${ }^{4}$ Y. F. Chen, Ph.D. thesis, Purdue University, 1984.

${ }^{5}$ Y. F. Chen, M. Dobrowolska, J. K. Furdyna, and S. Rodriguez, Phys. Rev. B 32, 890 (1985).

${ }^{6}$ E. U. Condon, Rev. Mod. Phys. 9, 432 (1937).

${ }^{7}$ E. I. Rashba and V. I. Sheka, Fiz. Tverd. Tela (Leningrad) 3, 1735 (1961) [Sov. Phys. —Solid State 3, 1257 (1961)]; 3, 1863
(1961) [3, 1357 (1961)].

${ }^{8}$ V. I. Sheka, Fiz. Tverd. Tela (Leningrad) 6, 3099 (1964) [Sov. Phys. - Solid State 6, 2470 (1965)].

${ }^{9}$ Y. Yafet, in Solid State Physics, edited by F. Seitz and D. Turnbull (Academic, New York, 1963), Vol. 14, p. 1.

${ }^{10}$ B. D. McCombe, Phys. Rev. 181, 1206 (1969).

${ }^{11}$ M. H. Weiler, R. L. Aggarwal, and B. Lax, Phys. Rev. B 17, 3269 (1978).

${ }^{12}$ M. Dobrowolska, A. Witowski, J. K. Furdyna, T. Ichiguchi, H. D. Drew, and P. A. Wolff, Phys. Rev. Lett. 49, 845 (1982); Phys. Rev. B 29, 6652 (1984). 